\title{
Message dissemination in VANET: protocols and performances
}

Anh Tuan Giang, Anthony Busson, Véronique Vèque

\subsection{INTRODUCTION OF VANET MESSAGE DISSEMINATION}

The most promising applications of Vehicular Ad Hoc NETworks (VANET) are safety applications. Embedded systems and sensors are becoming ubiquitous and more often found in our vehicles. Data exchanged by these systems help the driver to take appropriate decisions. These systems can inform the driver about a local anomaly, a too short inter-distance with the leading vehicle, help to adhere to road codes such as pavement marking, etc. Safety applications can be more efficient if information from these sensors is exchanged between neighboring vehicles. Communication between vehicles can also be used to alert the drivers about a dangerous situation, an accident for instance. As a result, a timely warning may help the driver to avoid an emergency stop or sometimes, a collision. Other applications, not directly linked to safety, as the dissemination of information about traffic conditions or even advertising are also promising and should appear quickly in our vehicles.

All safety applications suppose that exchanging messages which are disseminated to all or part of the vehicles come from an infrastructure or from the vehicles themselves. Data dissemination generally refers to the process of spreading data or information over distributed wireless networks. From the networking point of view, it requires broadcast capabilities at the link layer, allowing a frame to be transmitted to all the vehicles in the radio scope. It also supposes implementation of network and transport mechanisms to disseminate the message in the whole network. This dissemination uses one of the two available communication modes. The message will be disseminated in a multi-hop fashion when the vehicle-to-vehicle $(\mathrm{V} 2 \mathrm{~V})$ communication is enabled and will be broadcasted by all the Road Side Units (RSU) when infrastructure-to-vehicle (V2I-I2V) communications are used instead. A hybrid version is also possible, RSUs broadcast the messages and, as they do not cover the whole network, some vehicles are selected to forward the message in order to complete the dissemination. These messages can be flooded at a certain number of hops or in a given area (geocasting) depending on the application purposes. In $\mathrm{V} 2 \mathrm{~V}$ mode, the tasks of a dissemination protocol consist in selecting a pertinent set of vehicles to disseminate the message, and defining retransmission procedures to ensure the entire applications requirements on reliability, delay, etc. 
In this chapter, we present an overview and a performance evaluation of the existing mechanisms and protocols achieving message dissemination. We mainly focus on $\mathrm{V} 2 \mathrm{~V}$ communications. In the next section, we present how broadcast is performed at the link layer, supposing that the IEEE 802.11p standard [1] is used. We focus on this technology because it has been standardized since 2010 for vehicular communications and should equip all the vehicles in the near future. Dissemination protocol requirements are detailed in Section 1.3. Sections 1.4 and 1.5 present different basic mechanisms to disseminate broadcast messages used at higher layers. We also give some examples of dissemination protocols, and bring the focus to the dissemination protocols classification as proposed in the literature. We compare the performance of the different dissemination algorithms in Section 1.6. This evaluation is performed through simulations and takes into account different scenarios and models of traffic and radio environments. Finally, we conclude the chapter with Section 1.7.

\subsection{BROADCASTING IN IEEE STANDARD 802.11P}

In this section, we briefly present the IEEE 802.11 p standard [1]. We focus on broadcasting mechanisms: channel used to broadcast, transmission procedure, frame format and rules to access the wireless medium. It is noteworthy that wireless access mechanisms are detailed in the previous chapter. However in this chapter we highlight the differences between the services offered for unicast and broadcast frames. As we shall see, the service is really poorer for broadcast than for unicast functions.

\subsubsection{Channel}

As highlighted in the previous chapter, the FCC (Federal Communications Commission) allocated $75 \mathrm{MHz}$ of radio spectrum for DSRC (Dedicated Short Range Communication) [2]. The 5.9 GHZ DSRC spectrum is composed of six Service Channels $(\mathrm{SCH})$ and one Control Channel $(\mathrm{CCH})$. These channels are specified by the DSRC standard. Using these $10 \mathrm{MHz}$ channels, data rates of $3,4.5,6,9,12,18,24$, and $27 \mathrm{Mbps}$ are allowed including a preamble of $3 \mathrm{Mbp} / \mathrm{s}$ [2]. The modulation scheme used by DSRC is the Orthogonal Frequency Division Multiplexing (OFDM). The control channel is dedicated to broadcast frames for safety applications, service announcements, and vehicle-to-vehicle messages. It should be the preferred channel used to disseminate messages from safety and announcement applications. The other channels, the service channels, support both safety and user oriented applications, and could also be used to disseminate messages. 


\subsubsection{Transmission procedure}

The frame broadcasting transmission procedures (illustrated in Figure 1) are different in vehicle-to-vehicle and in infrastructure mode. When the vehicle-to-vehicle mode is used, the broadcast frame is directly sent by the source to the vehicles in the radio range. The destination address is then the MAC broadcast address (ff:ff:ff:ff:ff:ff). Vehicles in the radio range of this source receive the frame directly.

In the infrastructure mode, the $802.11 \mathrm{p}$ interface of a vehicle called On Board Unit (OBU), has to be associated with the Road Side Unit along the road. When the OBU intends to broadcast a frame, it sends it to the RSU, which in turn broadcasts it. The destination address is then set to the broadcast address. In order to guarantee that all the $802.11 \mathrm{p}$ interfaces in the transmitter radio scope receive the frame, the lowest available rate should be chosen to transmit the frame. This point is not specified in the standard but it is taken as granted in 802.11 technologies.

\subsubsection{Frame format}

Frame format in $802.11 \mathrm{p}$ is similar to that of 802.11 frames, and broadcast frames are identical to unicast frames. The only differences are the addresses. In Figure 2, we show the frame format for the two modes. In this figure, the MAC addresses correspond to the scenario in Figure 1. Frame 1 and 2 correspond to a broadcast transmitted from a vehicle in infrastructure mode. Frame 1 is the frame sent from the vehicle (OBU) to the RSU. The first address is the destination address, i.e. the RSU MAC address. The second address is the source address, the OBU MAC address. The third address is the broadcast address (ff:ff:ff:ff:ff). When the RSU broadcasts this frames, it permutes these addresses. The destination address becomes the broadcast address, the source address becomes the RSU MAC address and the third address becomes the OBU MAC address.

In vehicle-to-vehicle mode, the frame is directly broadcasted. The addresses are then the broadcast address and the MAC address of the source (OBU). For the third address, the OBU MAC address is reused as there is no OBU. 


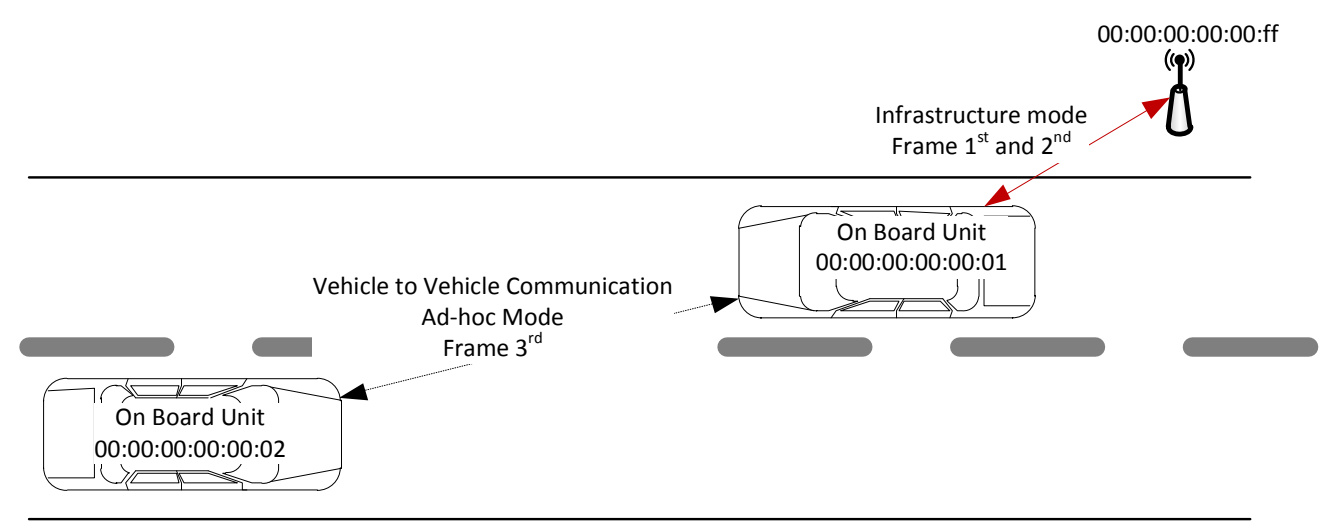

Figure 1 VANET operation mode: ad-hoc vs infrastructure

\begin{tabular}{|c|c|c|c|c|c|c|c|c|}
\hline bytes 2 & 2 & 6 & 6 & 6 & 2 & 6 & $0-2312$ & 4 \\
\hline \begin{tabular}{|l|} 
Frame \\
Control
\end{tabular} & $\begin{array}{l}\text { Duration } \\
\text { ID }\end{array}$ & 00:00:00:00:00:ff & 00:00:00:00:00:01 & ff:ff:ff:ff:ff:ff & $\begin{array}{c}\text { Sequence } \\
\text { Control }\end{array}$ & N/A & Frame Body & FCS \\
\hline
\end{tabular}

\begin{tabular}{|c|c|c|c|c|c|c|c|c|}
\hline bytes 2 & 2 & 6 & 6 & 6 & 2 & 6 & $0-2312$ & 4 \\
\hline \begin{tabular}{|l|} 
Frame \\
Control
\end{tabular} & $\begin{array}{l}\text { Duration } \\
\text { ID }\end{array}$ & ff:ff:ff:ff:ff:ff & 00:00:00:00:00:ff & 00:00:00:00:00:01 & \begin{tabular}{|c|} 
Sequence \\
Control
\end{tabular} & N/A & Frame Body & FCS \\
\hline & & & Frame 2: Broad & ast frame from $F$ & SU to OBI & & & \\
\hline bytes 2 & 2 & 6 & 6 & 6 & 2 & 6 & $0-2312$ & 4 \\
\hline $\begin{array}{l}\text { Frame } \\
\text { Control }\end{array}$ & $\begin{array}{l}\text { Duration } \\
\text { ID }\end{array}$ & ff:ff:ff:ff:ff:ff & 00:00:00:00:00:01 & 00:00:00:00:00:01 & \begin{tabular}{c|} 
Sequence \\
Control
\end{tabular} & N/A & Frame Body & FCS \\
\hline
\end{tabular}

Figure 2 Various frame formats.

The other fields of a frame are the Frame Control, Duration ID, Sequence Control and FCS (Frame Check Sequence). They have the same role as in the IEEE 802.11 family of protocols. The Frame Control field indicates the protocol version, the type of frame (data, management frame, acknowledgement, etc.), if it is a fragmented frame, if this frame is encrypted, and if the frame is sent to a RSU or to an OBU (infrastructure mode). The Duration ID field gives the transmission duration. The Sequence Control is the frame number and the FCS is a field used to detect transmission errors. The field following the Sequence Control is used to host a fourth address which is not used in our context. 


\subsubsection{Wireless transmission Acknowledgement}

In this sub-section, we describe the acknowledgment transmission procedure. It is noteworthy that wireless medium access, back-off and priority mechanisms have been detailed in the previous chapter. The reader can refer to the previous chapter for more information related to this topic.

A unicast transmission is systematically acknowledged from the receiver with a specific frame (an ACK). However, for a broadcasted frame, it is not practical to receive an ACK from each node receiving this frame. Indeed, if the receptions are acknowledged, each vehicle receiving the frame will send, almost at the same instant, an ACK back to the transmitting node.

This process may lead to a high collisions rate when multiple receivers coexist. This problem is known as the ACK explosion problem. Moreover, the sender is not supposed to have the list of the potential receivers. In the improbable case, where the sender knows the nodes/vehicles in its radio range, the use of ACK may be counter-productive.

In order to illustrate the problem, let us consider the following scenario. Assume that a vehicle is sending messages to 50 neighbors in its radio range. One of these vehicles is at the limit of the radio range and presents a high frame error rate (FER). When the sender sends its broadcast frame, it will be acknowledged 49 times. Since there is a missing ACK (from the vehicle with a high FER), the frame will be re-broadcasted again and again. Each time, there will be 49 receptions and 49 ACK until the $50^{\text {th }}$ vehicle receives the frame or the maximum number of transmissions is reached. This scenario may produce a lot of collisions and may waste network and OBU resources. Consequently, acknowledgment should not be permitted for broadcasted frames.

\subsubsection{Error detections and back-off}

As already stated, unicast and broadcast communications do not use same transmission procedures. The most important aspect that distinguishes the two communication modes is related to error detections. When a failure occurs, during the transmission of a broadcast frame, it is not detected by the transmitter because of lack of acknowledgement. Consequently, there is no retransmission in case of failure. Since the errors are undetectable, it is not possible to adapt the congestion window. If an important number of nodes are simultaneously contending for an access, it may result in a high number of collisions and cause a serious congestion. 
In the next Section, we present dissemination techniques used to disseminate messages to all the vehicles at several hops or in a certain geographic area. These mechanisms rely on the broadcast service offered by the IEEE $802.11 \mathrm{p}$, and must consequently compensate its lack of reliability.

\subsection{BROADCAST MESSAGE DISSEMINATION}

Through this chapter, we focus on dissemination of messages in the Vehicleto-Vehicle mode. The service offered by the layer 2 simply consists in broadcasting a frame to the nodes in the radio range of the sender, at one hop. In IEEE 802.11p, this service is unreliable. The sender does not know if its transmission has been received, and there is no retransmission in case of failure. However, safety applications rely on the dissemination of alert messages in a given area (limited by the number of hops or by geographical positions), not only at one hop. These messages are crucial as they contain important information on road safety. They need to be received by all the vehicles located in the area specified by the safety application. In other words, applications require a reliable dissemination of the messages. Delivery delay is also an important factor. Messages must be sent within the time specified by the application. Therefore, a protocol implemented at an upper layer is required to disseminate the message at several hops. This protocol must compensate the lack of reliability of the IEEE 802.11p and guarantee a fast and efficient delivery of the messages.

In the following, we introduce the basic mechanisms used to efficiently disseminate a message in classical ad hoc networks and VANETs. The first mechanism is the blind flooding. It is not suitable for VANET, but it allows us to explain the requirements of a good dissemination protocol. More efficient heuristics are then presented in Section 1.4 followed by VANET specific mechanisms in Section 1.5.

\subsubsection{Broadcast Storm problem}

There is a well-known problem in broadcasting in ad hoc networks, usually referring as Broadcast Storm. This issue was mentioned first in [3]. This problem happens if we use a basic flooding also called blind flooding to disseminate a packet in the network. Basic flooding works as follows. When a node receives a packet which has to be disseminated in the network, it checks if it is the first reception of this packet. If yes, it broadcasts it; otherwise it silently discards it. Since each node forwards the packet, it leads to an important redundancy. This redundancy depends on the network density: a node will receive as many packets as it has neighbors in its radio range. 
In Figure 3, we compare the number of transmissions and receptions with the blind flooding mechanism and an optimal broadcasting.

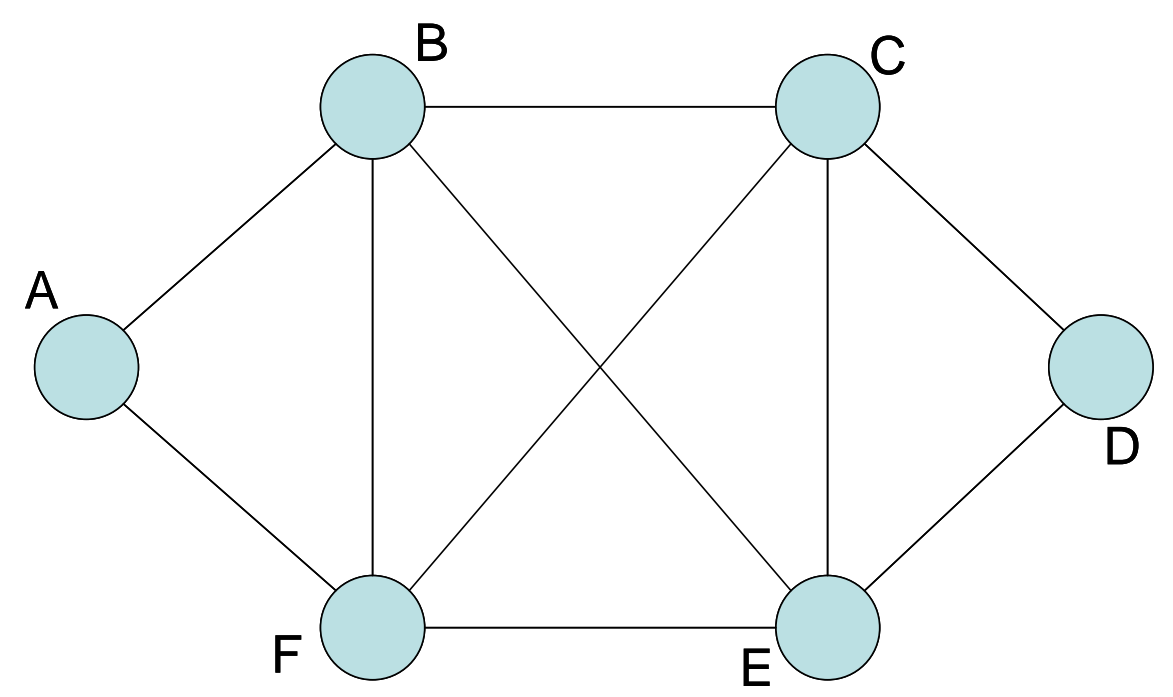

Figure 3. Example of topology of VANET

In Figure 3, the edges represent the wireless links between the nodes. We assume that node B wants to broadcast a message in the whole network. In the optimal case, we need only two broadcasts to reach all the nodes: B initially broadcasts the message and it is forwarded by $\mathrm{C}$. The transmission from $B$ reaches nodes $A, C, E$ and $F$. The transmission from $C$ reaches $D$. All the nodes have received the message with only two transmissions. In case of a blind flooding, each node transmits the message once. There are 6 transmissions and each node receives the message as many times as it has neighbors: 2 times for A and D, 4 times for B, C, F and E. It is the famous storm problem. In a VANET, a node may have up to 100 neighbors (the radio range of the IEEE $802.11 \mathrm{p}$ may reach up to $1 \mathrm{~km}$ and the density of vehicles may reach more than 100 vehicles per kilometer), such an approach will lead to 100 receptions for each vehicle. Such a scenario will significantly congest the network, causing packet transmissions to face heavy collisions, therefore wasting a lot of bandwidth and CPU resources.

\subsubsection{Dissemination protocol requirements}

A message will be broadcasted from the initial source through many intermediate nodes in order to cover the target area. This process is called multi-hop broadcast. Most of the existing dissemination protocols do not implement an acknowledgement and retransmission mechanism to ensure 
the good receptions of the messages. Such a mechanism would generate the same problems encountered at the layer 2: feedback explosion, etc. Consequently, the role of the dissemination protocol mainly boils down to the selection of a subset of nodes/vehicles. These vehicles will be in charge of forwarding the broadcast messages. Selection of these forwarders is a key challenge. They must form a connected network; otherwise the message could not reach all the forwarders and the targeted area. A certain level of redundancy is also required. In our context, the redundancy is the mean number of receptions per node for the same message. As broadcasts are unreliable, there is a subtle tradeoff between redundancy and reliability. If a node is in the radio range of only one forwarder, it will not receive the message at all if an error or collision occurs. It becomes dramatic if the receiver is also a forwarder. It is more adequate to have several forwarders in the radio range of each node to significantly increase the probability of reception and enhance the reliability. At the same time, this number must not be too important, as it will introduce a high number of receptions, generating congestion and bandwidth and OBU resources waste.

An appropriate protocol is thus required to ensure a good dissemination of the message. It is not possible to perform the optimal flooding, which minimizes the number of forwarders, because a complete and updated view of the topology is needed. This view requires a set of mechanisms not necessarily available: a link sensing mechanism allowing each node to discover its neighborhood, a link state routing protocol, etc. Moreover, even if such mechanisms are implemented in the VANET, it is not sure that routing information will be available for the dissemination protocol. As the dissemination requirements (reliability, delay, coverage, etc.) may be different from one application to another, the dissemination mechanism could be implemented at the application layer where the interaction with the routing layer is limited. Also, we have seen that the redundancy may be useful to increase the reliability. Therefore, instead of a protocol which minimizes the number of forwarders, pragmatic solutions guaranteeing a certain level of reliability, i.e. guarantee that most of the nodes will receive the message while keeping a low level of redundancy, are more adapted.

\subsection{DATA DISSEMINATION BASIC TECHNIQUES}

In this section, we present some basic solutions that aim at alleviating the storm problem. These solutions constitute the basic mechanisms used in more complex dissemination protocols. All these schemes are compared in Section 1.4. Probability, Counter, Distance and Location based Schemes have been first proposed in [3]. The other mechanisms have been proposed in different papers. References for these mechanisms are given in the paragraphs below. 


\subsubsection{Probability scheme}

This algorithm works as follows. When a node receives a message for the first time, it forwards/broadcasts it with probability $P$ with $0<P \leq 1$. For the next receptions, it silently discards the message. This simple mechanism limits the number of forwarders to a proportion $P$ of the nodes. Clearly, when $P=1$ this algorithm is equivalent to a blind flooding. But the choice of $P$ is not trivial. If $P$ is small, the dissemination may be stopped if the density of vehicle is not sufficient. If $P$ is great, the redundancy will be too important when the density of vehicles is high.

This scheme is not used in practice in VANET because it presents very poor performances as we shall see in Section 1.6.

\subsubsection{Counter-based scheme}

This algorithm assumes that after a message reception, the node has to wait for a while before its transmission. This delay is due to the back-off and MAC procedures or to a timer implemented by the protocol itself. Consequently, the node senses the medium while it is waiting for the messages sent by its neighbors and counts the number of times it receives the same message. At the end of the waiting time, the node rebroadcasts the message if it has received the message less than $k$ times and discards it otherwise; $k$ being a predefined threshold. The main benefit of this approach is that it bounds the number of transmissions and receptions whatever the vehicles density is (see [3] for more details). The value of $k$ may be chosen according to the aimed redundancy.

\subsubsection{Distance-based scheme}

This algorithm assumes that when a node receives a message, it is able to measure the distance to the transmitter. It can be simply obtained from a GPS (Global Positioning System) system. The position of the transmitter is then included in the message and the distance computed as the difference between the receiver and the transmitter locations. It can also be evaluated from the radio signal strength at the receiver. Examples of this solution are described in [4-5]. Let $d$ denote the distance separating the sender and the receiver. The node will forward the message if $d$ is greater than a predefined threshold $d_{\text {min }}$; and discards it otherwise. This scheme selects forwarders lying at a minimal distance of each others. It avoids retransmissions performed by nodes too close of each others, covering the same area and neighbors. The threshold $d_{\min }$ must be chosen in function of 
the radio range. A value close to the radio range will minimize the number of retransmissions. But, if there is no neighbor at a distance between $d_{\min }$ and the radio range, there will be no forwarder and the dissemination will fail.

\subsubsection{Location-based scheme}

As for the distance-based scheme, with this algorithm a node is supposed to know the distance of the neighbor from which it receives the message. With this distance, the node can calculate the additional area, also called additional coverage. This additional coverage is defined as the area of $A \backslash B$, where $\mathrm{A}$ is the region covered by the receiver, $\mathrm{B}$ the region covered by the transmitter, and $\mathrm{A} \backslash \mathrm{B}$ is the area of the set difference between $\mathrm{A}$ and $\mathrm{B}$ (region of $\mathrm{A}$ that does not belong to $\mathrm{B}$ ). If this additional coverage is greater than a predefined threshold, the node retransmits the message; otherwise it discards it. This scheme is very similar to the distance-based scheme in the context of VANET. The topology being linear, along a straight road, the additional coverage corresponds more or less to the distance. Moreover, the additional coverage is difficult to estimate in practice, since it depends on the radio environment (fading, shadowing, etc.) which is not known by the nodes. 


\subsubsection{Cluster-based scheme}

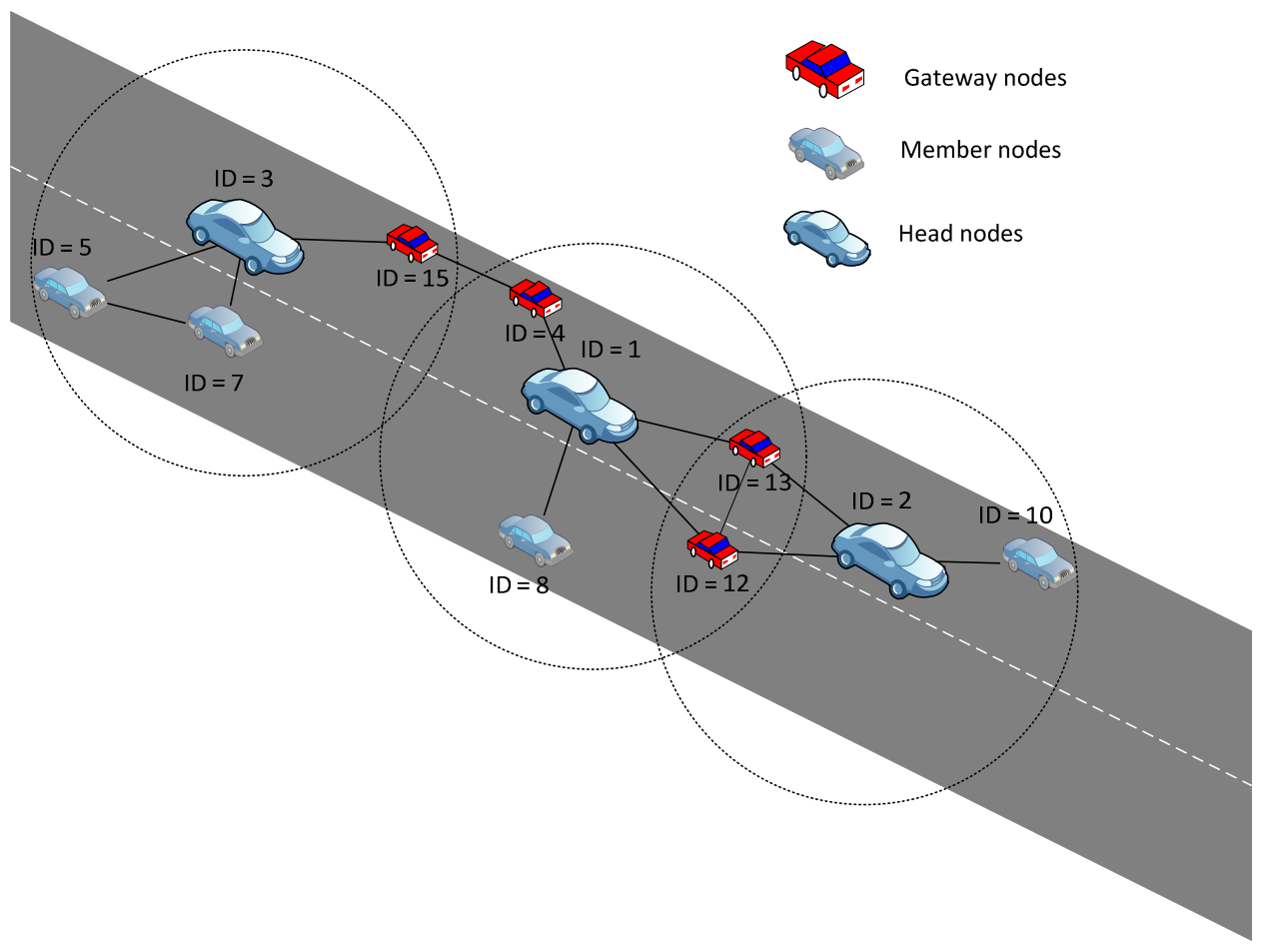

Figure 4 Clustered Networks: an Example.

With the cluster-based scheme, nodes are supposed to be divided into a set of clusters. A cluster is a subset of vehicles forming a convex network. Clusters are supposed to be disjoint, i.e. a node can belong to only one cluster. These clusters are used for different purposes: implement efficient broadcasts or create a hierarchy in the network allowing network protocol (particularly the routing protocols) to scale to any network size. A lot of clustering protocols have been proposed for ad hoc networks and VANETs. Generally, a node in a cluster is classified as head, gateway or member. The head, also called cluster-head, is a particular node used to build the cluster. There is only one head for each cluster and it is often at the core of its cluster. Gateways are the nodes sharing a link with another cluster. Members are the nodes which are neither heads nor gateways.

In this paragraph, we present an example of clustering algorithms based on the nodes ID [6] (Distributed Clustering Algorithm). More elaborated versions of this protocol have been proposed in [7]. Clusters are built as follows (an example is shown in Figure 4). Nodes periodically broadcast Hello messages in their radio range/neighborhood with their ID. If a node has the smallest ID among its neighbors, it becomes the cluster head. There is one cluster head for each cluster. The cluster is then identified by the ID 
of its cluster head. A node which is not a cluster head belongs to the same cluster as its neighbor with the smallest ID. Once the clusters are formed, each node specifies the ID of its head in the Hello messages. If a node detects that one of its neighbors is associated to a different cluster, it becomes a gateway. After forming the cluster, the algorithm for broadcasting will only allow the gateway or head using one of the beforementioned schemes: Probability Scheme, Counter-based Scheme, Distancebased Scheme, and Location-based Scheme, to retransmit messages while the member will be inhibited from broadcasting.

Specific solutions have also been proposed in the context of VANET and are presented in the next section.

\subsection{DISSEMINATION PROTOCOLS PROPOSED IN THE CONTEXT OF VANET}

Except some particular cases (Vehicular Information Broadcasting Relay [8] for instance), all of dissemination protocols use specific mechanisms to avoid the storm problem. In this Section, we classified the most representative and interesting dissemination protocols according to the basic mechanisms described earlier, and some mechanisms specific to VANET (Farthest node, Push based, and carry-and-forward schemes). All these protocols and mechanisms are then summarized in Table 1.

\subsubsection{Farthest node scheme}

With the revolution in new car generation, GPS becomes more and more popular. By using GPS, a vehicle can know its location and that of the transmitter (it can be included in the packet). Some dissemination protocols use this information to favor the farthest nodes from the previous emitter as the next forwarder. It maximizes the coverage area and minimizes the number of redundant receptions.

In [9-13], the farthest receiver is systematically the next forwarder, but the way it is selected is very different. In [10] (Directional Broadcast forwarding), each node is supposed to know its neighborhoods (IDs, and location of the vehicles in its radio range). A forwarder selects in its neighborhood the farthest node in the broadcast direction. A field in the message indicates the ID of the node responsible for the next retransmissions. In [9], [11] (Urban Multi-Hop Broadcast), [12] (Robust Message Dissemination), and [13] (Multi-Hop Vehicular Broadcast), when receiving a frame, a node triggers a retransmission timer (a black-burst in 
[11]; a deep presentation of black-burst techniques can be found in [14]) with a duration decreasing with the distance from the emitter. As a result, the farthest node retransmits first. Upon receiving this broadcast, the other nodes cancel their own transmission.

\subsubsection{Combination of probability based and farthest node schemes}

In [15-16], a vehicle retransmits the message according to a certain probability. This probability increases with the distance from the emitter and thus farther nodes are likely to be selected as forwarders. It is thus a combination of probability based and furthest node schemes.

In Smart Broadcast $[17,18]$, a dissemination scheme is implemented at the layer 2. When a node broadcasts a message, its radio range is divided into several zones. A contention window is associated to each zone. When a node in a given zone receives the message for the first time, it triggers a timer uniformly selected in the corresponding contention window. Values of the contention window are chosen to privilege retransmissions from the farthest vehicles. For example, the authors assume that the radio range is divided into three zones. Vehicles which are in the first zone, the closest from the transmitter, will use a contention window $C W=[16,31]$. For the second zone, in the middle, vehicles will use a contention window $\mathrm{CW}=[8,15]$, and $\mathrm{CW}=[0,7]$ for the third one. When a vehicle detects a retransmission from a vehicle downstream (farther with regard to the direction of the dissemination), it cancels its own retransmission. By doing so, vehicles in the farthest zone have a higher probability to retransmit the message first, and the other vehicles should cancel their transmissions. If there is no transmitter in the first zone, a vehicle in the second zone should be selected, and so on.

\subsubsection{Cluster-based scheme}

In [6-7, 19-21], authors propose different clustering algorithm. The classical clustering algorithm based on the cluster heads with highest degree is described in [6-7]. In [19] (Local Peer Group) and [20] (P2P Approach) roads or highways have been divided in logical sectors. A vehicle equipped with a GPS system is thus able to determine to which sector it belongs. All the vehicles in the same sector belong to the cluster. As clusters are formed with regard to pre-defined geographical zone and not from topological information, when the topology changes there is no need of Hello messages, update list of neighbors and cluster head. Authors of [19] also propose to use a fixed infrastructure, where the RSU transmits periodically the ID of their sector. In [21] (Application on Clustering), the authors 
propose to build the clusters with regard to the applications. For example, the cluster head of intersection assistance applications should be close to the targeted intersections.

\subsubsection{Push-based and pull-based mechanism}

Push-based data dissemination mechanisms use fixed RSU or moving vehicles to periodically deliver data messages to other vehicles. These messages are managed by data centers which collect data from applications and deliver it to the vehicles. A computer with a wireless interface or an info-station can play the role of data center. This type of mechanism is useful for applications which need to advertise information to a set of vehicles. For example, it may be an application which delivers information about road and traffic conditions, estimated time to reach destinations, etc. Also, it may be interesting to advertise commercial information about restaurants, gas stations, etc.

In [22], a push-based method named Data Pouring, is proposed. This protocol relies on data centers deployed along the road, intersections, etc. Data centers periodically broadcast information messages but do not cover the whole dissemination area. A dissemination mechanism using the vehicleto-vehicle mode completes the dissemination. This dissemination uses the farthest node mechanism.

Pull-based data dissemination mechanism is one form of request and response model. With this model, a vehicle sends query information to a specific location or target. For examples, it can inquire about a gas station, parking lot, or any other service. An example of such a protocol is detailed in [23] (Opportunistic Resource Exchange).

\subsubsection{Carry-store-forward mechanism}

It is worth noting that there are solutions which allow the dissemination even if there is no forwarder in the dissemination direction. An example is given in [24]. In the proposed mechanism, named Vehicle Assisted Data Delivery in VANET, a vehicle that needs to query data sends beacon message to acquire the list of its neighborhoods. Then, the carry-store-forward mechanism is used to deliver the data. The carry-store-forward technique consists for a vehicle in carrying the packet until it finds another vehicle in its neighborhood moving in the direction of the destination. Once this vehicle is found, it forwards the packet to this vehicle. A similar approach is also proposed in [25] (Mobility Centric Data Dissemination for VANET). 


\subsubsection{Summary}

In Table 1, we show the different protocols presented in this section. For each protocol, we indicate which basic mechanism is used, and if it requires a positioning system or a digital map. In the last column, we give the paragraph number where the protocol is described.

We do not compare the performance of these protocols. Indeed, they are difficult to classify because they have been proposed in different contexts (city, highway, etc.) and for different application requirements (delay, reliability, etc.). Moreover, some protocols are impossible to compare. For instance, protocols which use the carry-store-forward mechanism ([24] and [25] in the table) are useful and efficient only if the network is disconnected, in which case, lead to very significant delay that cannot be supported by certain applications.

Nevertheless, in order to give some insights on the performance of all these protocols, we evaluate and compare the basic mechanisms that they use. The results are presented in the next section. We shall show that the farthest node mechanism is the most efficient scheme to disseminate messages. It outperforms other schemes in terms of reliability (probability for a node of receiving the message), redundancy (mean number of times the same message is received by a node) and distance covered by the message. Moreover, the farthest node scheme does not require neighborhood information, and in its simplest form, can be implemented without any control messages.

Table 1 Messages dissemination protocols summarizations.

\begin{tabular}{|l|c|c|c|}
\hline \multicolumn{1}{|c|}{ Protocols } & Mechanism & $\begin{array}{c}\text { GPS - Digital } \\
\text { Map } \\
\text { Assumption }\end{array}$ & Paragraph \\
\hline $\begin{array}{l}\text { Vehicular } \\
\text { Information } \\
\text { Broadcasting Relay } \\
\text { [8] }\end{array}$ & Flooding & No & 1.5 \\
\hline $\begin{array}{l}\text { Directional } \\
\text { Broadcast } \\
\text { forwarding [10] }\end{array}$ & Farthest Node & Yes & 1.5 .1 \\
\hline $\begin{array}{l}\text { Urban Multi-Hop } \\
\text { Broadcast [11] }\end{array}$ & Farthest Node & Yes & 1.51 \\
\hline $\begin{array}{l}\text { Robust Message } \\
\text { Dissemination } \\
\text { RPB-MD [12] }\end{array}$ & Farthest Node & No & 1.5 .1 \\
\hline $\begin{array}{l}\text { Multi-Hop } \\
\text { Vehicular } \\
\text { Broadcast [13] }\end{array}$ & Farthest Node & Yes & 1.5 .1 \\
\hline
\end{tabular}




\begin{tabular}{|c|c|c|c|}
\hline $\begin{array}{ll}\text { Smart } & \text { Broadcast } \\
{[17-18]} & \\
\end{array}$ & $\begin{array}{c}\text { Farthest node - } \\
\text { Probability } \\
\end{array}$ & Yes & 1.5 .2 \\
\hline $\begin{array}{l}\text { Distributed } \\
\text { Clustering } \\
\text { Algorithm [6] }\end{array}$ & Clustering & No & 1.5 .3 \\
\hline $\begin{array}{l}\text { Local Peer Group } \\
\text { [19] }\end{array}$ & Clustering & Yes & 1.5 .3 \\
\hline P2P Approach [20] & Clustering & No & 1.5 .3 \\
\hline $\begin{array}{ll}\text { Application } & \text { on } \\
\text { Clustering [21] } & \\
\end{array}$ & Clustering & No & 1.5 .3 \\
\hline Data Pouring [22] & $\begin{array}{c}\text { Push-based } \\
\text { Farthest Node }\end{array}$ & Yes & 1.5 .4 \\
\hline $\begin{array}{l}\text { Opportunistic } \\
\text { Resource Exchange } \\
{[23]}\end{array}$ & Pull-based & Yes & 1.5 .4 \\
\hline $\begin{array}{l}\text { Vehicle Assisted } \\
\text { Data Delivery in } \\
\text { VANET [24] }\end{array}$ & $\begin{array}{c}\text { Carry-store- } \\
\text { forward }\end{array}$ & Yes & 1.5 .5 \\
\hline $\begin{array}{l}\text { Mobility Centric } \\
\text { Data Dissemination } \\
\text { for VANET [25] }\end{array}$ & $\begin{array}{c}\text { Carry-store- } \\
\text { forward }\end{array}$ & Yes & 1.5 .5 \\
\hline
\end{tabular}

\subsection{PERFORMANCE EVALUATION OF THE DISSEMINATION MECHANISMS}

In order to evaluate performance of the different basic mechanisms presented in the previous section, we have conducted various simulation runs that will be described in next sub-section. We consider the probabilistic, distance, counter, cluster-based and farthest node solutions. For the clusterbased scheme, we consider the clustering algorithm described in Section 1.4.5. Forwarding nodes in the cluster scheme use as well the counter-based scheme in order to limit the redundancy. For the counter based scheme, the counter is set to $2(k=2)$ : a node will retransmit the message if it receives less than 2 copies during a certain period. We do not present results on the location scheme as it is similar to the distance-based scheme. For the farthest node scheme, we suppose that at the first reception, the node triggers a timer. The timer duration is inversely proportional to the distance from the transmitter. Therefore, the timer of the farthest receiver will timeout first. At timer expiration, a node retransmits the message if it did not receive it from a node downstream (a second reception). In the ideal case, when a node forwards the message, all the receivers trigger their timer. The timer of the farthest receiver expires first, triggering its retransmission. All the previous receivers receive this message and cancel their own retransmissions. In our simulation runs, we vary the vehicle 
density, i.e. the mean number of vehicles per kilometer, from 5 vehicles $/ \mathrm{km}$ to 100 vehicles $/ \mathrm{km}$. The vehicle density variation allows us to study the algorithm behaviors for a wide range of traffic conditions, from very sparse to very dense.

In the next sub-sections, we detail the different elements of the simulators. Results are presented in sub-sections 1.6.6 to 1.6.8.

\subsubsection{Radio model}

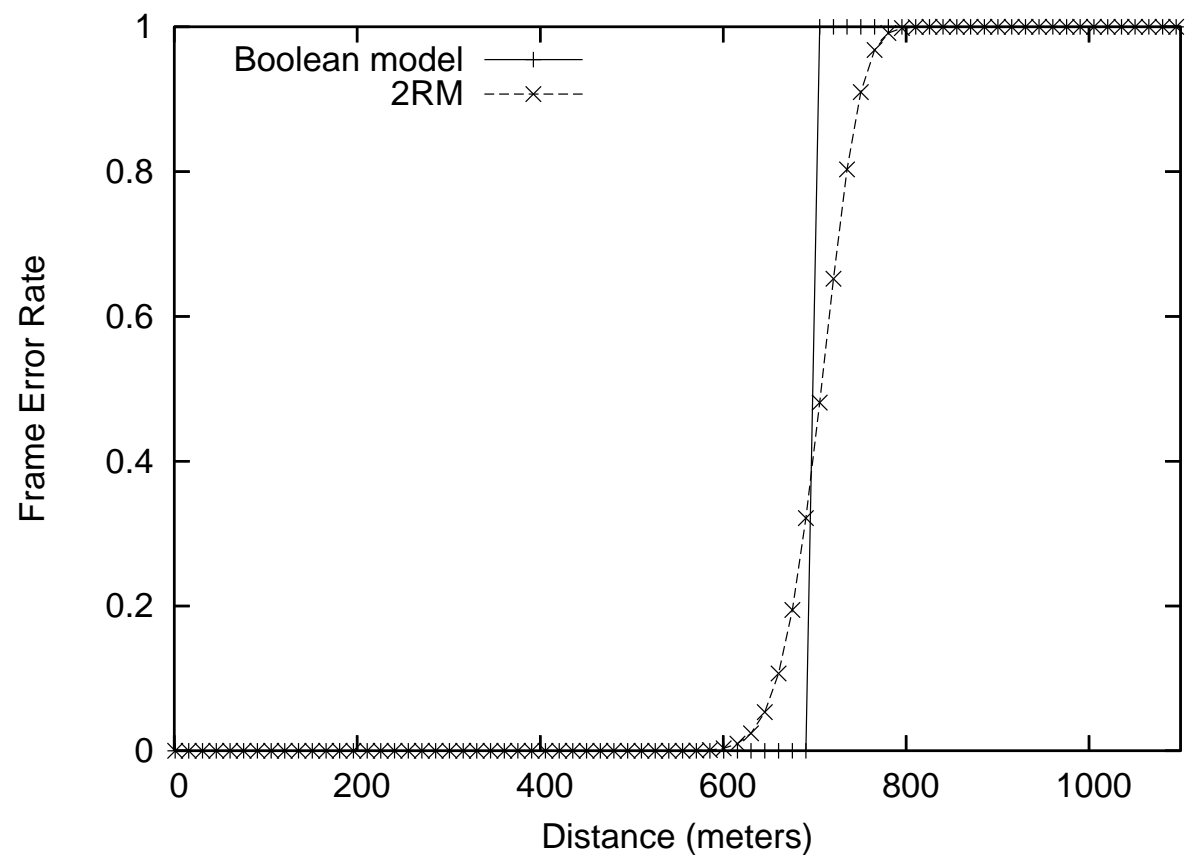

Figure 5 The two Frame Error Functions : Boolean and 2RM

The radio model used to determine when the frames are properly received by the vehicles consists in a FER function denoted $p(d)$. The latter gives the probability of loosing a frame with regards to the distance, $d$, between the transmitter and the receiver. In the simulations, we use this function to determine if a frame has been received or not. When a node transmits its frame, we compute for each potential receiver the distance $d$ to the transmitter. We draw a uniform random variable in the interval $[0,1]$ that we compare to $p(d)$. If the draw is less than $p(d)$, the frame is received properly, otherwise it is not received. Receptions are assumed to be independent of each others. We consider two different $F E R$ functions:

- The simplest one is the Boolean model. It is an ideal radio model where the radio range of a vehicle is a perfect ball. With this model, a frame will be received if the transmitter-receiver 
distance is less than a threshold $R$ (the radio range).

The FER function is then $p$ (distance) $=0$ if distance $<R$ and $p($ distance $)=1$ otherwise.

- In order to set the FER function $p$ (distance) according to the 802.11p standard, we consider a second model. We use the measurement based model developed in [26]. The proposed model is based on the two-ray path loss model referred as $2 R M$. The model takes into account wavelength of the $802.11 \mathrm{p}$ standard, heights, distances, gains of the two antennas (emitter and receiver) and frame length. Using the default parameters of the 802.11p standard listed in Table 2, we obtain the FER plot in Figure 5. The radio range obtained with this model is consistent with respect to the expected radio range of $802.11 \mathrm{p}$ in a rural environment (up to $1 \mathrm{~km}$ ).

Table 2 Simulator parameters.

\begin{tabular}{|l|l|}
\hline $\begin{array}{l}\text { Simulation } \\
\text { Parameters }\end{array}$ & $\begin{array}{l}\text { Numerical } \\
\text { values }\end{array}$ \\
\hline Frequency & $5.9 \mathrm{GHz}$ \\
\hline Transmission Rate & $3 \mathrm{Mbit} / \mathrm{s}$ \\
\hline Antenna heights & 1.5 meters \\
\hline Message length & 100 bytes \\
\hline
\end{tabular}

\subsubsection{Traffic model}

We consider three different types of road traffic. First, we suppose that intervehicles distances are constant. In the following figures, the inter-distance is then deduced from the traffic density $\mu$. The distance between two successive vehicles is given by $d_{\text {inter-distance }}=1 / \mu$.

In a second step, we take into account a more realistic model. We suppose that the distances between the vehicles are independent and follow an exponential distribution. It corresponds to the real distribution for low density of vehicles (see [28-29] for more details). The probability density function of the distance between two vehicles is then given by (for $d>0$ ):

$$
f_{\text {distance }}(d)=\mu \cdot e^{-\mu \cdot d}
$$

Assumptions about exponential distributions hold only for low traffic situations where drivers' behaviors are quite independent of each others. When the traffic density increases, this model is no more accurate. Interdistances between the vehicles become strongly dependent. Since it becomes difficult to model the traffic for such densities, we use a traffic simulator to generate realistic inter-distances. 


\subsubsection{Traffic simulator}

In order to obtain realistic vehicle movements, we have developed a traffic simulator. This traffic simulator allows us to faithfully emulate driver behavior. On a highway, driver behavior is limited to accelerating, braking or changing lanes. We assume that there is no off-ramp on the section of highway. A desired speed is associated to each vehicle. It corresponds to the speed that the driver would reach if he were alone in his lane. In the case the driver is alone (the downstream vehicle is sufficiently far), he adapts his acceleration to reach his desired speed (free flow regime). If he is not alone, he adapts his acceleration to the vehicles around (car following regime). He can also change lanes if the conditions of another lane seem better. All these decisions are functions of vehicles environment (speed and distance) and random variables used to introduce a different behavior for each vehicle. This kind of simulation is called micro simulation and the model we used is presented in detail in [27]. The model has been tuned and validated with regard to real traces observed on a highway [27]. We have simulated a road/highway of $5 \mathrm{~km}$ with 1, 2 and 3 lanes. The desired speed of vehicles follows a Normal distribution with mean $120 \mathrm{~km} / \mathrm{h}$ and standard deviation $\sigma=10$. The vehicles' density, shown in figures 6 to 9 , corresponds to the mean number of vehicles entering at the beginning of the simulated highway. When we considered several lanes, the density is divided by the number of lanes. The abscissa in the figures is then the sum of the densities on the different lanes.

\subsubsection{Timer}

We use different timers with regard to the dissemination protocol. A node waits for a certain time, defined by a timer function, before retransmitting the message. The retransmission can then be cancelled before the end of this timer, in the case of the counter-based or farthest node schemes for instance.

For the probabilistic, distance, and counter-based schemes the timer is equal to Timer $=50 \mathrm{~ms}+T$ where $T$ is a random variable uniformly distributed in the interval $[0,10 \mathrm{~ms}]$. The random variable $T$ is useful to avoid collisions: each potential forwarder schedules a different retransmission time.

For the farthest-node protocol, the timer function must decrease with the distance. We choose a function decreasing linearly with the distance to the transmitter, denoted $d$, and where the timer is at most $50 \mathrm{~ms}$, according to the following formula: $\operatorname{Timer}(d)=\left(-a^{*} d+b\right)^{*} 50$. With the chosen radio models, the maximum distance between the emitter and the receiver is about 800 meters. Consequently the parameters $a$ and $b$ become: $a=1 / 800$ and $b=1$. 


\subsubsection{Dissemination protocol simulator}

We have implemented another simulator, encoded in C, that simulates the different dissemination protocols. It implements the radio models and the two traffic models where the distance between the vehicles are constant or exponentially distributed. When the traffic simulator is used, trajectories are obtained through a pipe between the two simulators.

Once the distances between the vehicles are known, the different dissemination mechanisms are executed. The message is disseminated from a vehicle to all the vehicles $5 \mathrm{~km}$ upstream.

For each disseminated message, we collect different statistics: the covered distance, the probability of reception and the number of receptions. The covered distance is the distance between the message source and the farthest vehicle that receives the message. When the vehicles density is high, this distance is $5 \mathrm{~km}$ meaning that all the vehicles have received the message. The probability of reception corresponds to the proportion of nodes which have received the message. As for the number of receptions, we count the total number of receptions in the whole network that we divide by the number of nodes. It is thus the average number of received messages among all the nodes. All the statistics shown in the figures are the mean of 1000 samples. For each value of the density, we perform 1000 simulation runs and compute the average. A confident interval at $95 \%$ has been computed. But, with this number of simulations, error-bars are almost merged to the points and are not shown in order to keep the figures readable. 


\subsubsection{Impact of traffic on performances}

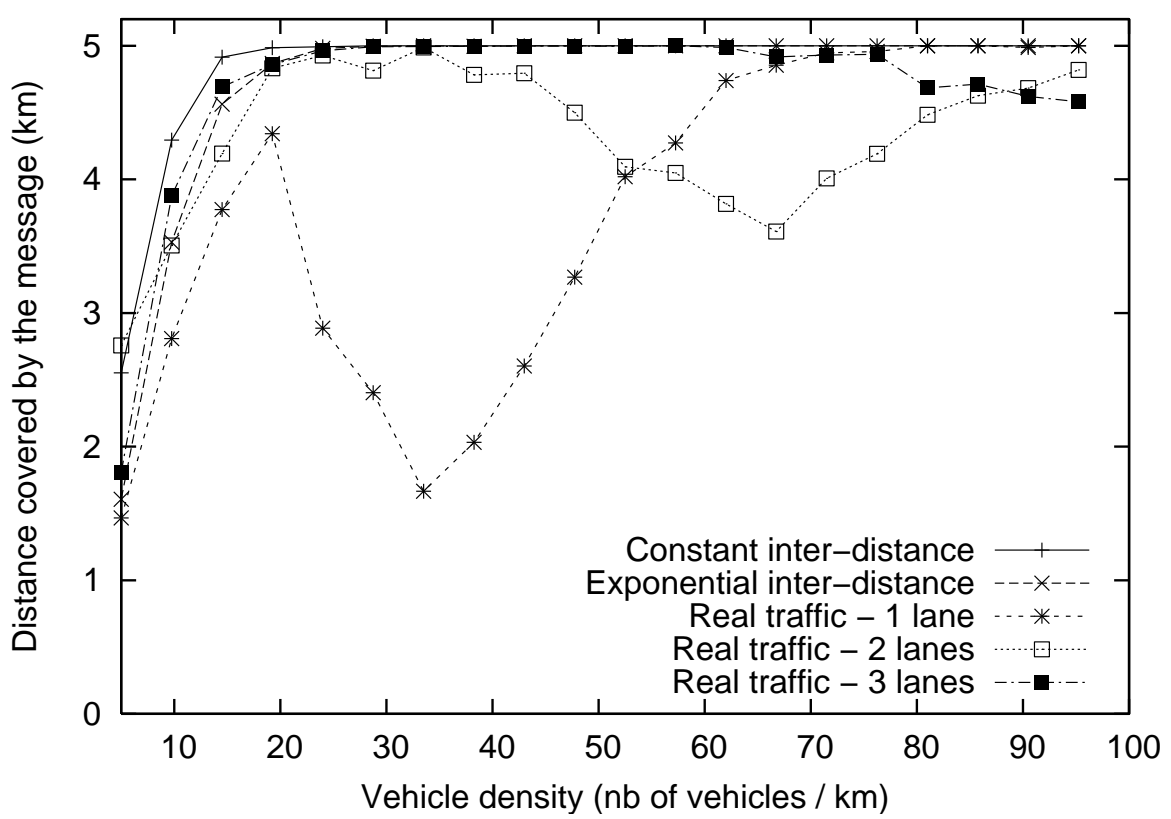

Figure 6 Impact of traffic on the performances: distance covered by the message for the probability-based scheme $(P=0.5)$ and the different traffic models. Highway length=5km. Radio model=Boolean.

In Figure 6, we plotted the distance covered by the message as function of the vehicles density. It varies from $5 \mathrm{veh} / \mathrm{km}$ to $100 \mathrm{veh} / \mathrm{km}$. We present the results for only one algorithm, the probability-based scheme, to highlight the impact of traffic nature on the performances. With the probabilitybased scheme, a vehicle rebroadcasts the message with a fixed probability $P$. In our simulations, we have set $P$ to 0.5 . Observations on the impact of traffic on the performances are similar for the other algorithms.

We observe that the distance covered by the message when the inter-distances between the vehicles are constant or exponentially distributed is merged except for small densities. Indeed, when the density is small, the exponential distribution may generate distances which are greater than the radio range. In this case, the message cannot be disseminated on the whole section since the network is disconnected. But the most interesting results are about the traffic simulator (Real traffic in the figure). We observe that when the density is about $30 \mathrm{veh} / \mathrm{km}$ and with a road/highway with 1 lane, the message covers only $1.8 \mathrm{~km}$ in average instead of the whole section $(5 \mathrm{~km})$. The same phenomenon appears, but is less important, for 2 and 3 lanes for densities equal to 68 and $95 \mathrm{veh} / \mathrm{km}$ respectively.

These behaviors can be explained by the nature of traffic. When the traffic 
reaches certain densities, most of the vehicles adapt their speed according to their environment (the other vehicles). It is known that under high vehicle densities [30-31], the traffic can be described in terms of different congestion phases: phases where the speeds of the vehicles are low and vary quite a lot between vehicles and phases where the vehicle speed is lower than with former phases with less variances between vehicles. This phenomenon explains the results obtained with the micro-simulator. When the density increases, the traffic goes through the different phases. The drops in the curves correspond to a phase where temporary jams occur (very dense sections with low speeds). It may just be caused by a vehicle slowing down, generating a wave effect upstream. A very sparse section of the highway then follows this jam. This phenomenon is often referred as stop-and-go traffic. In the next phase, thus for higher densities, the mean speed decreases but the vehicles are more homogeneously distributed on the road. When we observe vehicle densities on the simulated highway, we observe this phenomenon. When the density of vehicles entering in the simulator is high, we find sections of the highway with a lot of vehicles (up to 4-5 times the supposed density) corresponding to a jam, followed by sections with only a few vehicles. This difference is caused by local jams, and happens especially with one lane because it is very difficult to overtake slow vehicles.

It is clear that constant and exponential inter-distance are not suitable to model traffic (except for certain densities). Consequently, in the following, we only show the results from the traffic simulator. 


\subsubsection{Impact of radio models}
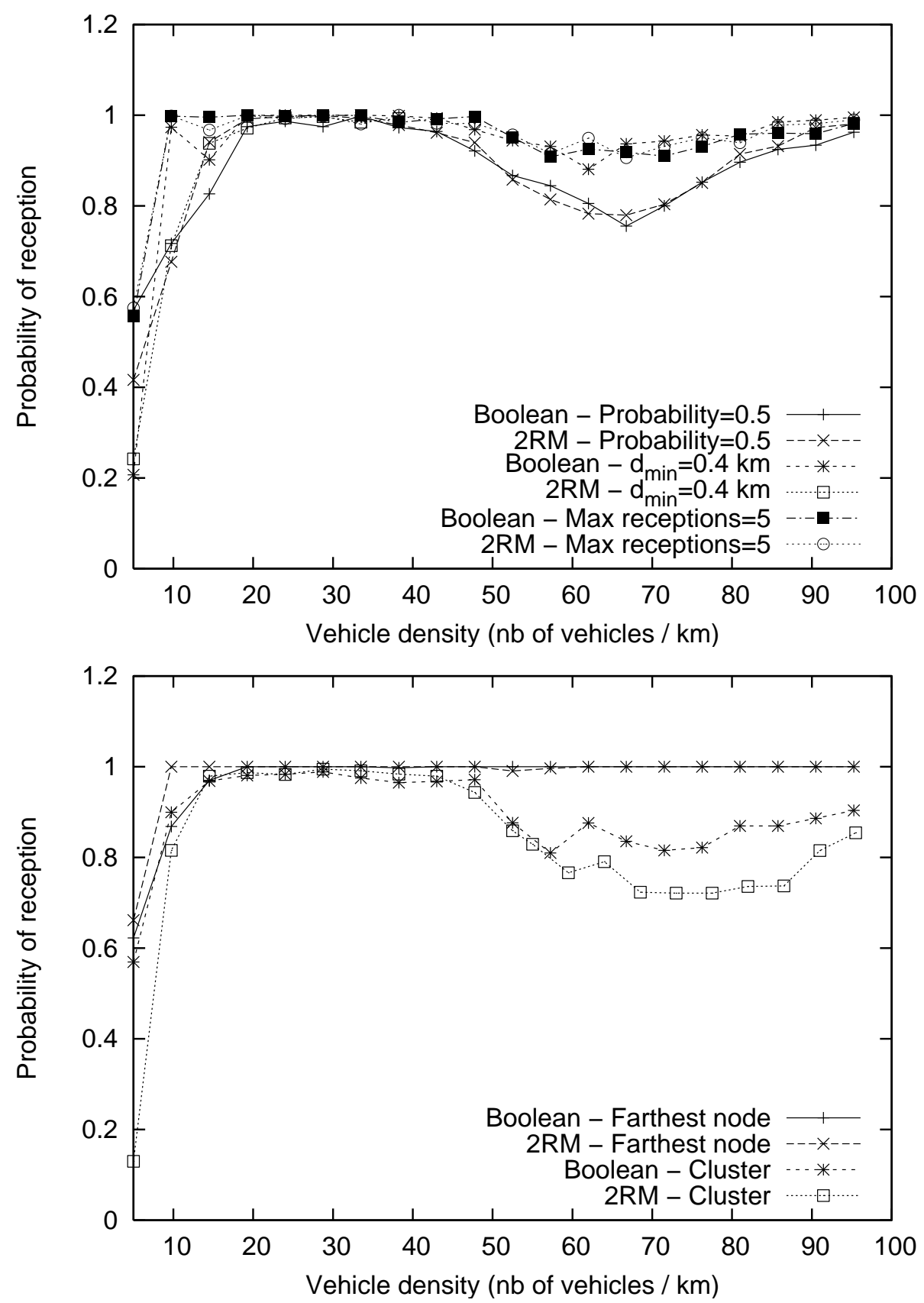

Figure 7. Impact of the radio models: probability of receptions. All the algorithms are considered. Vehicles trajectory are generated by the traffic simulator for a highway with length $5 \mathrm{~km}$ and 2 lanes.

In figure 7, we show the probability of receptions with regards to the density. Vehicles trajectories are generated by the traffic simulator for a highway 
length of $5 \mathrm{~km}$ and with 2 lanes. All the basic dissemination algorithms are considered: probability-based (with $P=0.5$ ), distance-based (with $d_{\text {min }}=0.4 \mathrm{~km}$ ), counter-based (with $k=5$ ), cluster-based and for the farthestnode solution. For each algorithm, we plotted the results for the two radio models: the ideal (Boolean) where the radio range is fixed, and the more realistic model $(2 R M)$. The probability of reception is close to 1 when the density is greater than $10 \mathrm{veh} / \mathrm{km}$. However, for certain densities (about $70 \mathrm{veh} / \mathrm{km}$ ), we observe a decoupling of the $2 R M$ curves from the Boolean model. For the Boolean model, the probability of reception stays close to 1 , but it drops significantly for the $2 R M$ model. For all algorithms there are only $80 \%$ of the nodes which receive the message. The only algorithm which is not impacted by the radio model is the farthest node solution where $100 \%$ of the nodes receive the message. The $2 R M$ model affects the results when the density becomes critical, i.e. when the traffic becomes very inhomogeneous. In this case, very dense traffics are followed by very sparse ones. For the sparse sections, we may have only a few vehicles ensuring the network connectivity. These few nodes might not forward the message: because they have cancelled their transmissions with probability 1-P in the probability-based scheme; because they are at distance less than $d_{\min }$ from the previous forwarder with the distance-based algorithm; or because they already received $k$ times the message from upstream vehicles in the counter-based scheme. For the cluster-based scheme, it is due to the low level of allowed redundancy: a gateway retransmits the message if it has not received it more than 2 times. This behavior also exists for the Boolean model but it is accentuated by the fact that in the $2 R M$ model transmission may fail.

It appears that the radio models have an important impact on the performances. For a realistic model, where the FER is not 0 or 1 , only the farthest node scheme stays efficient. In the following, we consider only the 2RM model. 


\subsubsection{Comparison between the algorithms}

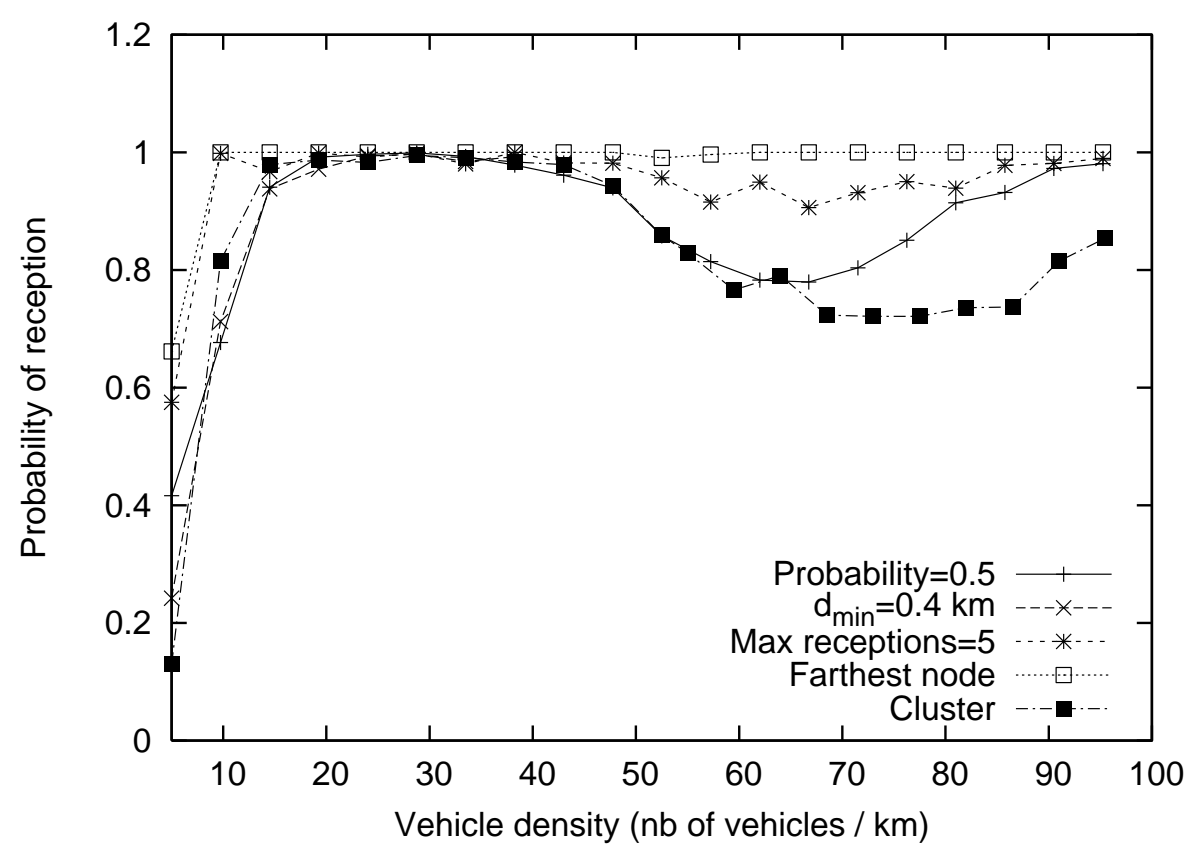

Figure 8. Comparison of the probability of receptions for the different algorithms. Radio model: $2 R M$. Highway length $=5 \mathrm{~km}$ with 2 lanes.

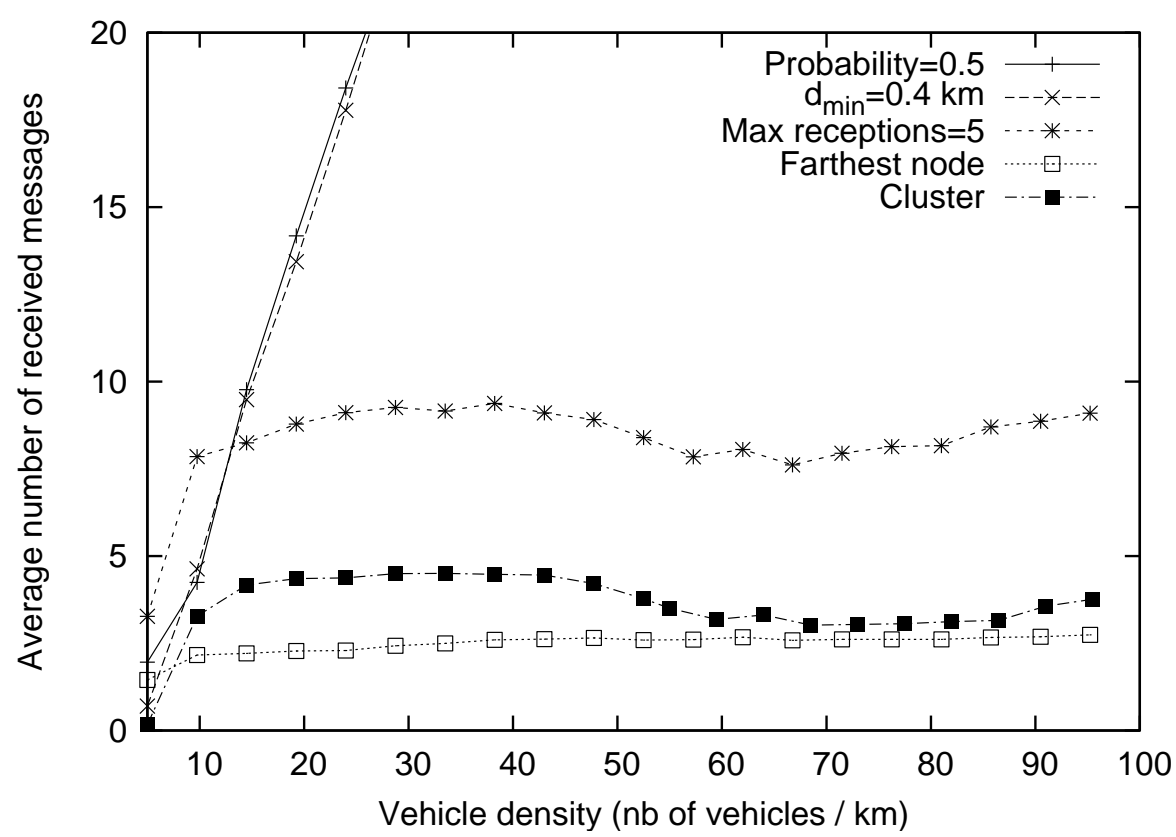

Figure 9. Comparison of the number of receptions for the different algorithms. Radio model: $2 R M$. Highway length $=5 \mathrm{~km}$ with 2 lanes.

In Figures 8 and 9, we plotted the probability of reception and the average 
number of received messages for the different algorithms in order to find out the scheme which offers the best tradeoff between both parameters. Obviously, the farthest node scheme outperforms other algorithms. Indeed, it keeps a probability of reception equals to 1 while minimizing the average number of received messages. The cluster-based scheme presents as well satisfying performances but the probability of receptions drop for high vehicle densities. Other schemes do not present acceptable results. In fact, their performances are strongly dependent of their parameter $(P, k$ and $d_{\text {min }) \text {. }}$

We suggest that these parameters should be efficiently tuned and adapted to vehicles density variations as the traffic is spatially inhomogeneous. For instance, $P$ should be small for high densities and high for small densities. However, even with such an approach the schemes in question do not behave as well as the cluster and farthest node schemes.

\subsection{CONCLUSION}

In this chapter, we proposed a survey of message dissemination techniques in VANET. First, we presented mechanisms used by the IEEE 802.11p standard to broadcast frames. We have shown that the service offered is very poor as congestion, collisions and errors may occur.

Mechanisms of the upper layers, in charge of disseminating the message several hops away, have to compensate for the lack of reliability of the link layer and must satisfy the application constraints in terms of delay or redundancy.

Classical ARQ (Automatic Repeat-reQuest) technique is not implemented at the upper layer as it leads to the same problems encountered at the link layer: difficulty to keep an up-to-date list of neighbors, feedback implosion, etc. Therefore, more pragmatic approaches should be used. These approaches rely on a certain redundancy to guarantee the reliability.

We have presented the basic mechanisms, used in most dissemination protocols to avoid the famous storm problem and discussed on their pertinence and applicability. We distinguished mechanisms proposed in general ad hoc networks to the ones specific to VANET. We listed dissemination protocols proposed in the literature. A classification of these protocols has been given with regard to these basic mechanisms.

We performed a number of simulations, considering different scenarios and models to compare efficiency of various dissemination protocols. The farthest node scheme was shown to be the most appropriate algorithm to disseminate messages in VANET. In fact, it adapts to different radio models and traffic situations while presenting low complexity and 
redundancy. We studied as well the impact of radio and road traffic models on the performance parameters: realistic models are required as they significantly impact the results.

\section{References}

1. IEEE Computer Society. (2011). Ieee standard for information technology telecommunications and information exchange between systems - local and metropolitan area networks - specific requirements - part 15.4 : Wireless medium access control (mac) and physical layer (phy) specifications - amendment 6 : Wireless acces in vehicular environments, Technical report, IEEE Computer Society.

2. ITS Standards Advisory: Dedicated Short Range Communications http://www.standards.its.dot.gov/Documents/advisories/dsrc_advisory.htm

3. Ni, S., Tseng, Y., Chen, Y. and Sheu, J. (1999). The broadcast storm problem in a mobile ad hoc network, Proc. ACM Mobicom, pp. 151-162.

4. Parker, R. and Valaee, S. (2007). Vehicular Node Localization Using Received-SignalStrength Indicator, IEEE Transactions on Vehicular Technology, 56(6), pp 3371-3380.

5. Elnahrawy, E., Li, X. and Martin, R.P. (2004) The limits of localization using signal strength: a comparative study, Proc. IEEE Communications Society Conference on Sensor and Ad Hoc Communications and Networks, IEEE SECON 2004.

6. Basagni, S. (1999). Distributed clustering for ad hoc networks, Proc. International Symposium on Parallel Architectures, Algorithms and Networks, I-SPAN 99.

7. Gerla, M. and Tzu-Chieh Tsai, T. (1995). Multicluster, mobile, multimedia radio network, Journal of Wireless Networks, 1(3), pp. 255-265.

8. Matsuda, S., Koike, H. and Okada, H. (2000). Vehicular information broadcasting relay (VIBROR) protocol for inter-vehicle-communications, Proc. 52nd IEEE Vehicular Technology Conference, VTC 2000.

9. Alshaer, H. and Horlait, E. (2004). Emerging client-server and ad-hoc approach in inter-vehicle communication platform, Proc. 60 ${ }^{\text {th }}$ IEEE Vehicular Technology Conference, VTC 2004.

10. Campelli, L., Cesana, M. and Fracchia, R. (2007). Directional broadcast forwarding of alarm messages in vanets, Proc. IEEE Conference on Wireless On-demand Network System, WONS 2007.

11. Korkmaz, G., Ekici, E. and Ozguner, F. (2004). Urban multi-hop broadcast protocol for inter-vehicle communication systems, Proc. 1st $^{\text {st }}$ international workshop on Vehicular ad hoc networks, VANET 2004.

12. Liu, C. and Chigan, C. (2008). RPB-MD: A novel Robust Message Dissemination Method for VANETs, Proc. IEEE Global Telecommunication Conference, Globecom 2008.

13. Osafune, T., Lin, L. and Lenardi, M. (2006). Multi-Hop Vehicular Broadcast (MHVB), Proc. International Conference on ITS Telecommunications, pp. 757-760.

14. Sobrinho, J. L. and Krisnakumar, A. S. (1999). Quality of service in ad-hoc carrier sense multiple access wireless networks, IEEE Journal on Selected Areas in Communications, 17, pp. 1353-1368.

15. Alshaer, H. and Horlait, E. (2005). An optimized adaptive broadcast scheme for intervehicle communication, Proc. 61 ${ }^{\text {st }}$ IEEE Vehicular Technology Conference, VTC 2005 Spring.

16. Chiasserini, C., Gaeta, R., Garetto, M., Gribaudo, M. and Sereno, M. (2006). Efficient broadcasting of safety messages in multi-hop vehicular networks. Proc. Parallel and Distributed Processing Symposium, IPDPS 2006. 
17. Chiasserini, C., Fasolo, E., Furiato, R., Gaeta, R., Garetto, M., Gribaudo, M., Sereno, M. and Zanella, A. (2005). Smart Broadcast of Warning Messages in Vehicular Ad Hoc Networks, Proc. Workshop Interno Progetto NEWCOM.

18. Fasolo, E., Zanella, A. and Zorzi, M. (2006). An Effective Broadcast Scheme for Alert Message Propagation in Vehicular Ad Hoc Networks, Proc. International Conference on Communication, ICC 2006.

19. Chen, W. and Cai, S. (2005). Ad hoc peer-to-peer network architecture for vehicle safety communications, IEEE Communications Magazine, 43, pp. 100-107.

20. Chisalita, I. and Shahmehri, N. (2002). A peer-to-peer approach to vehicular communication for the support of traffic safety applications, Proc. 5th International Conference on Intelligent Transportation System, pp. 336-341

21. Reumerman, H., Roggero, M. and Ruffini, M. (2005). The application-based clustering concept and requirements for intervehicle networks, IEEE Communications Magazine, 43, pp 108-113.

22. Zhao, J., Zhang, Y. and Cao, G. (2007). Data Pouring and Buffering on the Road: A New Data Dissemination Paradigm for Vehicular Ad Hoc Networks, IEEE Transaction on Vehicular Technology, 56 (6), pp. 3266-3276.

23. Xu, B., Ouksel, A. and Wolfson, O. (2004). Opportunistic resource exchange in intervehicle ad hoc networks, Proc. IEEE International Conference on Mobile Data Management.

24. Zhao, J. and Cao, G. (2008). VADD: Vehicle-assisted data delivery in vehicular ad hoc networks, IEEE Transaction on Vehicular Technology, 57 (3), pp. 1910-1922.

25. Wu, H., Fujimoto, R., Guensler, R. and Hunter, M. (2004). MDDV: a mobility-centric data dissemination algorithm for vehicular networks, Proc. ACM International Workshop on Vehicular Ad Hoc Networks, VANET 2004.

26. Barsocchi, P., Oligeri, G. and Potortì, F. (2007). Frame error model in rural Wi-Fi networks. Proc. International Symposium on Modeling and Optimization.

27. Ahmed, K. I. (1999). Modeling Drivers' Acceleration and Lane Changing Behavior, PhD thesis, Massachusetts Institute of Technology.

28. Gazis, D.C. (2002) Traffic Theory. (Kluwer Academic Publishers).

29. Mannering, F. L., Washburn, S. and Kilareski, W. P. (2008) Principles of highway engineering and traffic analysis, 4th Edition. (Wiley).

30. Nagatani, T. (2002). The physics of traffic jams, Journal of Reports on progress in physics, 65(9), pp 1331-1386.

31. Abul Magd, A. Y. (2007) Modelling highway-traffic headway distributions using superstatistics, Physical review, 76 (2). 